\title{
Community-Acquired Pneumonia in Children: Myths and Facts
}

\author{
Ki Wook Yun, MD, PhD ${ }^{1,2}$ Rebecca Wallihan, $\mathrm{MD}^{3} \quad$ Alexis Juergensen, MS ${ }^{1}$ \\ Asuncion Mejias, MD, $\mathrm{PhD}^{1,3}$ Octavio Ramilo, $\mathrm{MD}^{1,3}$ \\ ${ }^{1}$ Center for Vaccines and Immunity, The Research Institute at \\ Nationwide Children's Hospital, Columbus, Ohio \\ ${ }^{2}$ Division of Infectious Diseases, Department of Pediatrics, Seoul \\ National University College of Medicine, Seoul, Korea \\ ${ }^{3}$ Division of Infectious Diseases, Nationwide Children's Hospital, \\ Address for correspondence Octavio Ramilo, MD, Division of \\ Infectious Diseases, Nationwide Children's Hospital, 700 Children's \\ Drive, Columbus, $\mathrm{OH} 43205$ \\ (e-mail: octavio.ramilo@nationwidechildrens.org).
} Columbus, Ohio

Am J Perinatol 2019;36(suppl S2):S54-S57.

\begin{abstract}
Keywords

- community-acquired pneumonia

- children

- transcriptional profiles

- biomarkers

Community-acquired pneumonia (CAP) is the leading cause of death in children $<5$ years of age worldwide. It is also one of the most frequent infectious diseases in children, leading to large antibiotic use and hospitalization even in the industrialized countries. However, the optimal management of CAP in children is still not well defined. Currently, respiratory viruses are considered the most frequent etiologic agents, but detection of viruses in the upper respiratory tract does not guarantee causation of pneumonia, nor precludes the presence of a bacterial pathogen. In both the upper and lower respiratory tract, respiratory viruses and pathogenic bacteria interact. Emerging evidence indicates that dual viral-bacterial infections function synergistically in many cases and together likely enhance the severity of CAP. Therefore, new and advanced technologies capable of sensitively and specifically discriminating viral, bacterial, and viral-bacterial coinfections are needed. Instead of focusing on the pathogen, analysis of host immune transcriptome profiles from children with CAP can potentially offer diagnostic signatures, help to assess disease severity, and eventually, prognostic indicators. An optimized management strategy by using molecular pathogen testing and transcriptome profiling will facilitate prompt, more appropriate, and targeted therapies, which in turn will lead to improved clinical outcomes in children with CAP.
\end{abstract}

Pneumonia is the leading cause of death in children $<5$ years of age worldwide. Estimated numbers of deaths by pneumonia were 0.762 million and the cause-specific mortality rate was 5.455 cases per 1000 livebirths in 2015 . $^{1}$ Although mortality rates do not reach the levels observed in low- and middleincome countries, the morbidity and financial burden associated with pneumonia remains significant also in developed countries. In a recent large epidemiologic study in the United States, the annual incidence of community-acquired pneumonia (CAP) requiring hospitalization was 15.7 cases per 10,000 children, with the highest rate among children younger than 2 years of age. ${ }^{2}$

Despite the fact that pneumonia is a remarkably common disease yielding an enormous impact on childhood health, antibiotic resistance, and direct and indirect health care costs, there are some diagnostic and therapeutic dilemmas that remain unresolved, and the optimal management of pneumonia in children is still not well defined. Furthermore, there is no validated scoring system to predict which children with pneumonia should be hospitalized or how to objectively assess the disease severity.

\section{Etiologic Agents}

Although the specific etiologic agent is not identified in many cases of CAP in children, respiratory viruses are detected in more than half of cases. In a prospective, multicenter, population-based, active-surveillance study to identify the
Copyright (c) 2019 by Thieme Medical Publishers, Inc., 333 Seventh Avenue, New York, NY 10001, USA. Tel: +1(212) 584-4662. ISSN 0735-1631. 
etiology of CAP in children (Etiology of Pneumonia in the Community [EPIC] study by the Centers for Diseases Control and Prevention), viruses were detected in $66.2 \%$ of children with CAP requiring hospitalization. ${ }^{2}$ Respiratory syncytial virus (RSV), rhinovirus (RV), and human metapneumovirus (hMPV) were the viruses most commonly detected in 28.0, 27.3 , and $12.8 \%$ of cases, respectively.

Pyogenic bacteria are detected in a relatively small proportion of CAP in children, but their early identification is critical, as they can cause severe and/or complicated pneumonia and even mortality. Pyogenic bacteria were identified as the responsible pathogens causing pneumonia in $7.3 \%$ of children in the EPIC study. Streptococcus pneumoniae (3.6\%) was the most common, followed by Staphylococcus aureus (1.0\%) and Streptococcus pyogenes (0.7\%). Mycoplasma pneumoniae, an atypical bacterial pathogen, was detected in $8.0 \%$ of children with CAP, particularly in those 5 years of age or older. ${ }^{2}$

We conducted a prospective, multicenter, observational cohort study for CAP in children (Children's Hospital's Initiative for Research in Pneumonia [CHIRP] study) during 2015 to 2018. This study enrolled 441 children aged 2 months through 18 years with CAP diagnosed from six referral hospitals in Ohio, United States, and included outpatients (13.8\%) as well as inpatients (86.2\%). In the preliminary data analysis, viruses were detected in $55.6 \%$ of children, pyogenic bacteria in $3.6 \%$, and atypical bacteria in $8.8 \%$. The proportion of viruses and bacteria detected was comparable to those of the EPIC study.

Another recent and large study on pneumonia in children, the Pneumonia Etiology Research for Child Health (PERCH) study, was designed to determine the cause of severe pneumonia in 4,232 children aged 1 to 59 months living in developing areas. Epidemiologic and etiologic findings from the PERCH study varied from other CAP studies on children in developed countries. ${ }^{3}$

\section{Viral-Bacterial Interaction}

Viral-bacterial coinfections were detected in 7.0 and $3.9 \%$ of children with CAP in the EPIC and CHIRP studies, respectively. However, these proportions likely represent low estimates because of the low sensitivity of current assays (e.g., blood culture) for bacterial identification. In the EPIC study, compared with children infected with viruses alone, those with virus-bacterial coinfections had a higher frequency of leukocytosis, consolidation on chest radiography, parapneumonic effusions, intensive care unit admission, need for mechanical ventilation, and an increased length of hospital stay. ${ }^{4}$ Particularly, RSV and influenza not only contribute to the seasonal pattern of invasive pneumococcal disease (IPD) but also influenced the severity and outcome of patients with IPD. ${ }^{5,6}$

Studies in animal models revealed that lung inflammation, rate of bacteremia, and mortality were increased in mice coinfected with RSV and S. pneumoniae when compared with mice with single infections. ${ }^{7}$ We recently performed a case-control study to detect potentially pathogenic bacteria (PPB), such as S. pneumoniae, Moraxella catarrhalis, and Haemophilus influenzae in nasopharyngeal samples from children $<2$ years of age with RSV bronchiolitis compared with age- matched healthy controls. Detection of each PPB was more frequent in children with RSV infection than in healthy controls. Moreover, detection of S. pneumoniae and H. influenzae in RSV-infected children was associated with enhanced disease severity. These data suggest that pathogenic bacteria detected in the upper airway during acute RSV infection may play a role in disease pathogenesis and severity. ${ }^{8}$

\section{Diagnostic Challenges}

The diagnosis of pneumonia could be established in most cases by clinical features and chest radiograph findings, although there is substantial overlap with bronchiolitis in infants. However, reliable single tests for identifying the specific pathogen (or pathogens) causing pneumonia do not exist, although an accurate and rapid etiologic diagnosis will result in improved care with focused antimicrobial therapy, fewer unnecessary tests and procedures, and potentially shorter hospital stays in children with CAP. ${ }^{9}$

Children with atypical bacterial pneumonia could be presented with clinical features, as well as laboratory and radiographic findings that are similar to those of viral pneumonia. Even in children with pyogenic bacterial pneumonia, the initial clinical findings can be similar to viral pneumonia, such as upper respiratory tract symptoms, low level of inflammatory markers, and interstitial infiltrates on chest radiograph. ${ }^{10} \mathrm{C}$ reactive protein and procalcitonin may assist to assess response to therapy in patients with more serious pneumonia cases, but identification of a cutoff that is both sensitive and specific for defining bacterial etiologies remains challenging. ${ }^{9}$

Another problem with the etiologic diagnosis of pneumonia in children is the difficulty in obtaining adequate specimens for pathogen detection. Because the primary site of the infection is the lung, high yield specimens would have to be obtained from lung tissue or alveolar fluid. However, procedures like lung tap and bronchoalveolar lavage are very invasive, and not routinely performed as standard of care in children with CAP. Gram stain and sputum culture are also infrequently performed because of the difficulty of obtaining these samples in young children. Pleural fluid analyses help to establish the etiologic diagnosis of pneumonia, but most of the CAPs are not accompanied by enough pleural fluid to be aspirated and a pleural tap is another invasive technique that can induce complications. Therefore, blood culture, that has limited sensitivity, is essentially the sole method to detect pyogenic bacteria in children with CAP. In addition, because respiratory viral infections appear to predispose to bacterial superinfections, a respiratory virus identified in a child with CAP does not necessarily exclude the presence of a bacterial pathogen. ${ }^{9}$ This commonly leads to inappropriate interventions and overly cautious treatment with excessive use of antibiotics and unnecessary hospitalizations.

The diagnosis of $M$. pneumoniae is frequently made by polymerase chain reaction (PCR)-based testing. Although studies have questioned the diagnostic value of this assay, ${ }^{11}$ the EPIC and CHIRP studies confirmed its utility in various clinical settings. For respiratory viruses, real-time PCR of a nasopharyngeal swab or aspirate specimen is usually used in children. 
However, some respiratory viruses detected by PCR in children with CAP have been also detected in asymptomatic children. Therefore, detection of respiratory viruses from the upper respiratory tract may not reveal causation of pneumonia, particularly with RV or adenovirus, which are shed for prolonged time and/or may reactivate in the context of other disease processes. On the other hand, RSV, influenza, and hMPV are rarely detected in asymptomatic healthy children. ${ }^{12}$

Nevertheless, identification of respiratory viruses in the context of CAP in children may have clinical implications, and antibacterial therapy may be held in the absence of clinical, laboratory, or radiographic findings that suggest a bacterial coinfection. ${ }^{10}$ In the CHIRP study, children with no bacteria identified and positive viral testing were more likely to be discharged without antibiotic therapy. ${ }^{13}$

\section{Can We Apply Transcriptomics to CAP in Children?}

By shifting the focus from the infectious pathogen, application of host gene profiles by transcriptomics can potentially identify diagnostic signatures, markers of disease severity, and, eventually, prognostic indicators in the various clinical settings. ${ }^{14}$ Blood is an attractive source of clinically relevant information. It serves as a reservoir for immune cells that migrate to and from the site of infection, and are exposed to factors released in the bloodstream as a result of the infection. Different classes of pathogens trigger specific pattern-recognition receptors differentially expressed on immune cells and elicit distinct immune responses. Using microarray or RNAseq technology, we can measure the differences in gene expression patterns present in blood immune cells as induced by various types of infectious agents. Therefore, transcriptional profiles of peripheral blood leukocytes could help discriminating viral, bacterial, and viral-bacterial coinfections in patients with pneumonia. A recent study ${ }^{15}$ performed in adults with pneumonia revealed that transcriptional profiling was superior to procalcitonin in distinguishing bacterial from viral lower respiratory tract infections.

Molecular distance to health (MDTH) is a novel, objective, and unbiased metric, which summarizes the global transcriptional perturbation of each individual patient sample compared with healthy controls into a single numeric score. In children hospitalized with CAP, MDTH scores measured within 24 hours of hospitalization were independently associated with a longer duration of hospitalization, regardless of the pathogen detected. This suggests that transcriptional biomarkers such as MDTH may also help classify children with CAP by disease severity and determine the need for hospitalization or intensive care. ${ }^{16}$

\section{Intervention Strategies}

Evidenced-based guidelines for the management of infants and children with CAP were developed by the Pediatric Infectious Diseases Society and the Infectious Diseases Society of America in 2011. ${ }^{9}$ As mentioned above, the difficulties to exclude bacterial infections in children with
CAP, precludes making a definitive diagnosis, and dilemmas in managing CAP in children are still present.

Influenza vaccination may reduce pneumococcal pneumonia as well as influenza-associated pneumonia possibly suggesting that these pathogens have an additive or synergistic effect in the pathogenesis of pneumonia. ${ }^{9}$ A pivotal randomized clinical trial in children showed that pneumococcal immunization was associated with significant reductions in hospitalizations caused by viral-bacterial pneumonia. ${ }^{17}$ Therefore, both influenza and pneumococcal vaccinations represent important opportunities for preventing or at least attenuating CAP severity in children.

\section{Conclusion}

In summary, viral testing in conjunction with clinical, laboratory, and radiographic findings may provide guidance of antibiotic use for CAP in children. Host transcriptome profiles may prove to have superior sensitivity for the identification of specific pathogen and may enable the identification of the viral-bacteria coinfections. Optimizing the management of these children will also lead to decreased use of antibiotics and potentially play an important role in hindering antibiotic resistance, lowering health care costs, and reducing the number of adverse events associated with antibiotic use. In addition, an accurate classification of patients based on disease severity from the very beginning by the application of novel biomarkers such as MDTH will provide prompt and targeted therapies, which in turn will lead to improved clinical outcomes.

\section{Conflict of Interest}

A.M. has received research grants from NIH and Janssen; fees for participation in advisory boards from Janssen, Roche; and fees for lectures from Abbvie. O.R. has received research grants from Janssen, NIH, the Bill \& Melinda Gates Foundation and the Ohio Children's Hospital Association (OCHA); fees for participation in advisory boards from Merck, Sanofi, and Medimmune; and fees for lectures from Pfizer.

\section{References}

1 Liu L, Oza S, Hogan D, et al. Global, regional, and national causes of under-5 mortality in 2000-15: an updated systematic analysis with implications for the Sustainable Development Goals. Lancet 2016;388(10063):3027-3035

2 Jain S, Williams DJ, Arnold SR, et al; CDC EPIC Study Team. Community-acquired pneumonia requiring hospitalization among U.S. children. N Engl J Med 2015;372(09):835-845

3 O'Brien KL, Baggett HC, Brooks WA, et al. Introduction to the epidemiologic considerations, analytic methods, and foundational results from the pneumonia etiology research for child health study. Clin Infect Dis 2017;64(Suppl 3):S179-S184

4 Nolan VG, Arnold SR, Bramley AM, et al. Etiology and impact of coinfections in children hospitalized with community-acquired pneumonia. J Infect Dis 2018;218(02):179-188

5 Techasaensiri B, Techasaensiri C, Mejías A, McCracken GH Jr, Ramilo O. Viral coinfections in children with invasive pneumococcal disease. Pediatr Infect Dis J 2010;29(06):519-523 
6 O’Brien KL, Walters MI, Sellman J, et al. Severe pneumococcal pneumonia in previously healthy children: the role of preceding influenza infection. Clin Infect Dis 2000;30(05):784-789

7 Jordan-Villegas A, Chang ML, Khokhar S, et al. Respiratory Syncytial Virus (RSV) Infection Predisposes Mice to Severe Pneumococcal Disease and Bacteremia. Infectious Diseases Society of America 48th Annual Meeting; October 21-24 2010. Vancouver, British Columbia, Canada

8 Diaz A, Garcia-Maurino C, Naples J, et al. Nasopharyngeal bacterial detection in children with RSV infection: impact on clinical outcomes. IDWeek; October 3-7 2018. San Francisco, CA

9 Bradley JS, Byington CL, Shah SS, et al; Pediatric Infectious Diseases Society and the Infectious Diseases Society of America. The management of community-acquired pneumonia in infants and children older than 3 months of age: clinical practice guidelines by the Pediatric Infectious Diseases Society and the Infectious Diseases Society of America. Clin Infect Dis 2011;53(07):e25-e76

10 Virkki R, Juven T, Rikalainen H, Svedström E, Mertsola J, Ruuskanen O. Differentiation of bacterial and viral pneumonia in children. Thorax 2002;57(05):438-441

11 Spuesens EB, Fraaij PL, Visser EG, et al. Carriage of Mycoplasma pneumoniae in the upper respiratory tract of symptomatic and asymptomatic children: an observational study. PLoS Med 2013; 10(05):e1001444
12 Self WH, Williams DJ, Zhu Y, et al. Respiratory viral detection in children and adults: comparing asymptomatic controls and patients with community-acquired pneumonia. J Infect Dis 2016;213(04):584-591

13 Marzec S, Ambroggio L, Desai A, et al for the Children's Hospital Initiative for Research in Pneumonia (CHIRP). Impact of viral testing on duration of antibiotic therapy in children hospitalized with community acquired pneumonia (CAP) in a multicenter study. 2018 Pediatric Academic Societies Annual Meeting; May 5-8 2018. Toronto, Canada.

14 Ramilo 0, Mejías A. Shifting the paradigm: host gene signatures for diagnosis of infectious diseases. Cell Host Microbe 2009;6(03): 199-200

15 Suarez NM, Bunsow E, Falsey AR, Walsh EE, Mejias A, Ramilo O. Superiority of transcriptional profiling over procalcitonin for distinguishing bacterial from viral lower respiratory tract infections in hospitalized adults. J Infect Dis 2015;212(02):213-222

16 Wallihan RG, Suárez NM, Cohen DM, et al. Molecular distance to health transcriptional score and disease severity in children hospitalized with community-acquired pneumonia. Front Cell Infect Microbiol 2018;8:382. Doi: 10.3389/fcimb.2018.00382

17 Madhi SA, Klugman KP; Vaccine Trialist Group. A role for Streptococcus pneumoniae in virus-associated pneumonia. Nat Med 2004;10(08):811-813 